\title{
Prognostischer Wert für Asthma bronchiale mäßig
}

\author{
Die bronchiale Hyperreagibilität (BHR) ist ein wichtiges, \\ allerdings nicht sehr spezifisches Charakteristikum bei Asthma. \\ Daher lässt eine BHR im Kindesalter auch nicht unbedingt \\ darauf schließen, dass sich in den nächsten Jahren ein Asthma \\ entwickelt, wie A. Riiser et al. zeigen konnten. \\ Am J Respir Crit Care Med 2012; 186: 493-500
}

Aus einer Norwegischen Geburtskohorte evaluierten die Autoren bei insgesamt 530 Kinder im Alter von 10 Jahren das Vorliegen von Asthma und einer BHR. Zur Beurteilung der BHR diente den Autoren die Metacholin-Dosis, die eine $20 \%$ ige Reduktion des Einsekundenvolumens $\mathrm{FEV}_{1}$ $\left(\mathrm{PD}_{20}\right)$ im Metacholin-Test hervorgerufen hatte. Außerdem untersuchten sie die belastungsabhängige Bronchokonstriktion (EIB: exercise-induced bronchoconstriction), indem sie die Reduktion des $\mathrm{FEV}_{1}$ nach einem standardisierten Ergometertest hoher Intensität maßen. Dieselben Kinder wurden 6 Jahre später erneut untersucht.

Ein aktives Asthma lag vor, wenn mindestens 2 der folgenden Kriterien erfüllt waren: Die ärztliche Diagnose eines Asthmas, Asthmasymptome oder eine Asthmabehandlung im vergangenen Jahr. In der Allgemeinbevölkerung Norwegens liegt die Prävalenz eines aktiven Asthmas mit 16 Jahren bei $13,7 \%$, in der hier untersuchten Kohorte bei $18 \%$. Dabei hatten 74\% der Kinder, die bereits mit 10 Jahren ein aktives Asthma aufwiesen, dieses mit 16 Jahren immer noch, während $10 \%$ der Kinder ohne Asthma im Alter von 10 Jahren bis zum 16. Lebensjahr ein aktives Asthma neu entwickelt hatten.

\section{Einfluss nachweisbar, aber gering $\nabla$}

Im Alter von 10 Jahren wiesen $51 \%$ der Kinder mit einer $\mathrm{PD}_{20} \leq 8 \mu \mathrm{mol}$ Methacholin und auch $51 \%$ der Kinder mit EIB ein aktives Asthma, ein Asthma in Remission oder Asthmasymptome auf. Über die Hälfte (54\%) der Kinder mit einer ausge- prägten BHR im Alter von 10 Jahren hatten mit 16 Jahren ein aktives Asthma.

Der Grad von $\mathrm{PD}_{20}$ und EIB im Alter von 10 Jahren erhöhte das Risiko für das Vorliegen eines Asthmas 6 Jahre später. Pro $\mu \mathrm{mol}$ Methacholin erhöhte sich das Asthmarisiko um $6 \%$ ( $\beta=0,94$; 95\%-Konfidenzintervall [KI] $0,92-0,96]$, pro \% $\mathrm{FEV}_{1}$-Reduktion nach dem Standardbelastungstest um mehr als $10 \%(\beta=1,10$; 95\%-KI 1,06-1,15). Die Fläche unter der Kurve war für $\mathrm{PD}_{20}$ größer als für $\mathrm{EIB}$ $(0,69$; $95 \%-\mathrm{KI} 0,62-0,75$ versus 0,60 ; $95 \%-$ KI $0,53-0,67)$.

Diese Einzelergebnisse erklären nur 10 und 7\% der Varianz. Auch zusammen genommen sind sie nur für 14\% der Varianz zwischen der Asthmaprävalenz im Alter von 16 Jahren im Vergleich zur Situation 6 Jahre früher verantwortlich zu machen. Mit abnehmender $\mathrm{PD}_{20}$ und ansteigender EIB im Alter von 10 Jahren nimmt die Vorhersagewahrscheinlichkeit für die Entwicklung eines Asthma s im Alter von 16 Jahren aber zu.

\section{Fazit}

Eine BHR im Alter von 10 Jahren ist ein deutlicher, wenn auch nur mäßiger Prädiktor für ein aktives Asthma 6 Jahre später, so die Autoren. Der Methacholin-Test ist dabei aussagekräftiger als der Belastungstest. Andere wesentliche Einflussfaktoren für die Asthmaprädiktion waren ein aktives Asthma bereits mit 10 Jahren und das Geschlecht: Für Jungen ließ sich eine sicherere Prognose stellen als für Mädchen.

Friederike Klein, München

\section{Putzjob: Chemikalien bergen Asthmarisiko}

Der Arbeitsplatz kann das Risiko für die Entwicklung von Asthma bei Erwachsenen erhöhen, wie die Allergologin Rebecca Ghosh vom Imperial College London und ihre Kollegen nachgewiesen haben. Sie fanden bei 1 von 6 Probanden einen Zusammenhang zwischen einer Atemwegserkrankung und dem Job. Die Forscher konnten 18 Berufe ausmachen, die das Asthmarisiko potenziell erhöhen. Menschen, die Reinigungstätigkeiten nachgehen, sind besonders betroffen. Denn diese Gruppe ist gesundheitsbelastenden Chemikalien ausgesetzt. „Obstruktive Atemwegserkrankungen können neben vielen unterschiedlichen Faktoren aus dem persönlichen Umfeld auch durch Einwirkungen verursacht werden, die bei der Arbeit auftreten", sagt Elke Biesel, Sprecherin der Deutschen Gesetzlichen Unfallversicherung im Spitzenverband der Berufsgenossenschaften und Unfallversicherungsträger der öffentlichen Hand. Die Forscher gehen davon aus, dass etwa $16 \%$ der Menschen, die in den 1950erJahren geboren wurden, im Erwachsenenalter durch ihren Job an Asthma erkrankt sind. „Eine Umfrage zeigt, dass ein Viertel mit Asthma bereits ernste berufliche Konsequenzen wegen ihrer Erkrankung hinnehmen mussten; die Hälfte der Befragten fühlt sich durch das Asthma in ihrer Leistungsfähigkeit bei der Arbeit eingeschränkt", sagt Andreas Hellmann, Vorsitzender des Bundesverbands der Pneumologen in Deutschland. Staub, trockene Atemluft oder Schimmelsporen könnten Symptome hervorrufen oder die Erkrankung verschlimmern.

Nach einer Mitteilung der pressetext Nachrichtenagentur $\mathrm{GmbH}$

Erratum

Fälschlicherweise wurden auf der Titelseite der Februar-Ausgabe 12 CMEPunkte abgedruckt. Wie aus dem Fortund Weiterbildungsbeitrag im Heft hervorgeht, können durch die Beantwortung der Fragen nur maximal 3 CME-Punkte gesammelt werden. Wir bitten, diesen Fehler zu entschuldigen. 